\title{
A RADIO SEARCH FOR PULSAR COMPANIONS TO SLOAN DIGITAL SKY SURVEY LOW-MASS WHITE DWARFS
}

\author{
Marcel A. Agüeros ${ }^{1,5}$, Fernando Camilo ${ }^{1}$, Nicole M. Silvestri ${ }^{2}$, S. J. Kleinman ${ }^{3}$, Scott F. Anderson ${ }^{2}$, and \\ JAMES W. LIEBERT ${ }^{4}$ \\ ${ }^{1}$ Columbia Astrophysics Laboratory, Columbia University, New York, NY 10027, USA; marcel@ astro.columbia.edu \\ 2 Department of Astronomy, University of Washington, Seattle, WA 98195, USA \\ ${ }^{3}$ Gemini Observatory, Northern Operations Center, Hilo, HI 96720, USA \\ ${ }^{4}$ Steward Observatory, University of Arizona, Tucson, AZ 85721, USA \\ Received 2008 November 26; accepted 2009 March 4; published 2009 May 1
}

\begin{abstract}
We have conducted a search for pulsar companions to 15 low-mass white dwarfs (LMWDs; $M<0.4 M_{\odot}$ ) at $820 \mathrm{MHz}$ with the NRAO Green Bank Telescope (GBT). These LMWDs were spectroscopically identified in the Sloan Digital Sky Survey (SDSS), and do not show the photometric excess or spectroscopic signature associated with a companion in their discovery data. However, LMWDs are believed to evolve in binary systems and to have either a more massive white dwarf (WD) or a neutron star (NS) as a companion. Indeed, evolutionary models of low-mass X-ray binaries, the precursors of millisecond pulsars (MSPs), produce significant numbers of LMWDs, suggesting that the SDSS LMWDs may have NS companions. No convincing pulsar signal is detected in our data. This is consistent with the findings of van Leeuwen et al., who conducted a GBT search for radio pulsations at $340 \mathrm{MHz}$ from unseen companions to eight SDSS WDs (five are still considered LMWDs; the three others are now classified as "ordinary" WDs). We discuss the constraints our nondetections place on the probability $P_{\text {MSP }}$ that the companion to a given LMWD is a radio pulsar in the context of the luminosity and acceleration limits of our search; we find that $P_{\mathrm{MSP}}<10_{-2}^{+4} \%$.
\end{abstract}

Key words: pulsars: general - white dwarfs

Online-only material: color figure

\section{INTRODUCTION}

The number of known white dwarfs (WDs) has increased dramatically since the beginning of the Sloan Digital Sky Survey (SDSS; York et al. 2000). The most recent SDSS WD catalog, collected from its Data Release 4 (DR4; Adelman-McCarthy et al. 2006), yielded 9300 spectroscopically confirmed WDs, of which 6000 are new discoveries (Eisenstein et al. 2006). Model fits to the SDSS spectra find that the majority of these objects are hydrogen (DA) WDs for which the mass distribution peaks at the expected value of $0.6 M_{\odot} \cdot{ }^{6}$ But among the new SDSS WDs there are also a handful of candidate very low-mass WDs (LMWDs) with $M<0.4 M_{\odot}$. Such objects are noteworthy not only because of their rarity but because the Galaxy is not thought to be old enough to produce LMWDs through singlestar evolution.

The youngest WDs in the oldest Milky Way globular clusters have masses of $\sim 0.5 M_{\odot}$ (Hansen et al. 2007). Lower mass WDs must therefore undergo significant mass loss, presumably because they form in close binaries whose evolution includes a phase of mass transfer. During this phase, much of the WD progenitor's envelope is removed, stunting the WD's evolution by preventing a helium flash in its core and resulting in the observed low-mass, helium-core WD. ${ }^{7}$ As Marsh et al. (1995) put it, "low-mass WDs need friends." In examining seven WDs

\footnotetext{
5 NSF Astronomy and Astrophysics Postdoctoral Fellow.

6 The existence of this peak in the field DA mass distribution has been known for close to $30 \mathrm{yr}$; see Koester et al. (1979).

7 Kilic et al. (2007a) argue that an LMWD may form from the evolution of a single, metal-rich star. However, they predict that the binary fraction for WDs with $M \sim 0.4 M_{\odot}$ is $50 \%$ and rises to $100 \%$ for $M<0.2 M_{\odot}$. Thus, even in this scenario, the likelihood of finding companions to the SDSS LMWDs remains high.
}

with $M \leqslant 0.45 M_{\odot}$, they found that four are in doubledegenerate close binary systems, with orbital periods on the order of a few hours to a few days; for a fifth they identified a companion but were unable to confirm its nature or obtain an orbital period.

Subsequent studies of large samples of DAs found that roughly $10 \%$ have masses $\lesssim 0.4 M_{\odot}$, and that in the majority of these binaries the companion is likely to be a degenerate star (Liebert et al. 2004, 2005). The Marsh et al. (1995) systems are WD/WD binaries, but the LMWD companions can also be neutron stars (NSs; e.g., discussion in Driebe et al. 1998). In these systems, binary evolution produces an LMWD in a near-circular orbit around the NS "reborn" as a millisecond pulsar (MSP; for discussion of different evolutionary scenarios involving NSs, see Tauris \& van den Heuvel 2006).

Observationally, roughly $5 \%$ of field radio pulsars reside in binary systems. Most of these pulsars are MSPs, and in most cases their companions are thought to be LMWDs with $0.1 \lesssim M \lesssim 0.4 M_{\odot}$. Of the 52 MSP companions identified outside of globular clusters, Manchester et al. (2005) list 17 with masses $\lesssim 0.2 \quad M_{\odot}$, assuming that the systems have a median inclination of $60^{\circ}$. However, the MSP companions are frequently too faint for optical spectroscopy to confirm that they are LMWDs. Despite the observational challenges, there are several well-known MSP/LMWD systems, and in fact the majority of known He-core WDs are MSP companions (Panei et al. 2007); see van Kerkwijk et al. (2005) for a review of these systems.

That said, the birthrate of MSPs in the Galactic disk is thought to be $\sim 3 \times 10^{-6} \mathrm{yr}^{-1}$ (Lorimer 2008). Estimating the number of LMWDs in the Galaxy is difficult (e.g., discussion in Liebert et al. 2005), and the SDSS sample is best considered as providing a lower limit (because WDs are generally not explicitly targeted 
for SDSS spectroscopy, it is unclear how best to correct for, e.g., incompleteness). However, Liebert et al. (2005) used the Palomar Green Survey to estimate the formation rate of LMWDs to be $0.4 \times 10^{-13} \mathrm{pc}^{-3} \mathrm{yr}^{-1}$, implying that these make up about $10 \%$ of all DA WDs. ${ }^{8}$ Naively, this suggests that the LMWD birthrate in the volume sampled by the SDSS DR4 $\left(\sim 4 \mathrm{kpc}^{3}\right)$ is $\sim 2 \times 10^{-4} \mathrm{yr}^{-1}$, or nearly 2 orders of magnitude higher than that of MSPs. This discrepancy in the (admittedly very rough) birthrates suggests that there is a low likelihood of detecting MSP companions to SDSS LMWDs.

Still, because of the tantalizing theoretical and observational connections between LMWDs and MSPs, we searched for radio pulsations from putative pulsar companions to 15 spectroscopically confirmed SDSS LMWDs using the NRAO Green Bank Telescope (GBT). We describe the sample of SDSS LMWDs in Section 2 and our $820 \mathrm{MHz}$ observations in Section 3. We discuss the significance of our nondetections in Section 4 and conclude in Section 5.

\section{THE TARGETS: SDSS LOW-MASS WDS}

Our sample of candidate LMWDs was selected from the two SDSS WD catalogs (for a full description of the SDSS WD selection, WD models, and the autof it program used to obtain estimates for WD temperatures and masses, see Kleinman et al. 2004; Eisenstein et al. 2006). Two, SDSS J105611.03+653631.5 and J123410.37-022802.9, are described in detail in Liebert et al. (2004). An additional 11 candidate LMWDs were later proposed by Eisenstein et al. (2006). These WDs are selected to have temperatures $T_{\text {eff }}<30,000 \mathrm{~K}$, surface gravities (log $g$ ) more than $2 \sigma$ below 7.2, and $g<20 \mathrm{mag}$. None of these WDs show the photometric excess or spectroscopic signature associated with a companion in their discovery data (see, for example, Figures 21 and 22 in Eisenstein et al. 2006), as expected if the companion is a compact object.

Re-analysis of the SDSS spectra by Kilic et al. (2007b) confirmed that the Eisenstein et al. (2006) candidates are LMWDs in all but one case: they find that SDSS J204949.78+000547.3 is an A2 star. ${ }^{9}$ This leaves us with a sample of 12 LMWDs from the SDSS catalogs. To these, we add three LMWD candidates identified by Kleinman et al. (2004) that are present in the Eisenstein et al. (2006) WD catalog but are not classified by the latter authors as LMWDs. SDSS J131033.25+644032.8 and J234536.47-010204.9 are not included in the Eisenstein et al. (2006) LMWD list because they have $T_{\text {eff }}=$ 39,700 and $30,300 \mathrm{~K}$, respectively. The model fits for SDSS $\mathrm{J} 163800.36+004717.7$ return $T_{\text {eff }}=73,000 \mathrm{~K}$ and a $\log g$ that is only $1 \sigma$ below 7.2. Model fits to very cool and very hot WDs are somewhat unreliable, ${ }^{10}$ but we wish to be as inclusive as possible in assembling our sample. Adding these three gives us just 15 LMWD candidates spectroscopically identified in a survey volume of $\sim 4 \mathrm{kpc}^{3}$.

Figure 1 gives $\log g$ versus $T_{\text {eff }}$ for all of the candidate LMWDs as reported by Eisenstein et al. (2006). We use the models in Figure 1 to estimate $M_{\mathrm{WD}}$ for each of our LMWDs; their properties are presented in Table 1. While the masses of these WDs are comparable to those of LMWDs detected as

\footnotetext{
8 This contribution is $\sim 10 \times$ larger than indicated by the relative space densities of low-mass and ordinary $\left(0.6 M_{\odot}\right)$ WDs as it accounts for the presence of degenerate companions to the LMWDs.

9 This work was done after we had observed J2049+0005 and we therefore analyzed these data as well.

10 For example, differences between SDSS-derived temperatures and those in the literature for previously cataloged WDs are about $10 \%$ at $50,000 \mathrm{~K}$ (Eisenstein et al. 2006).
}

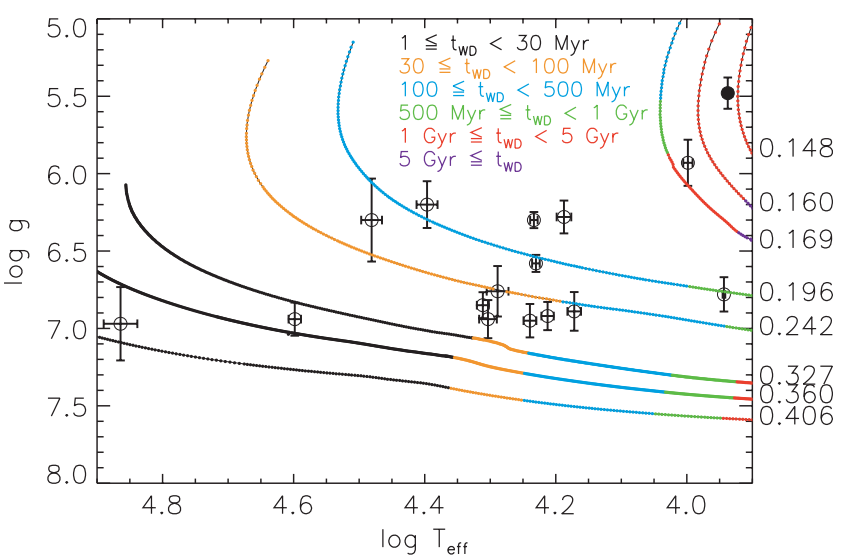

Figure 1. $\log g$ vs. $\log T_{\text {eff }}$ for the SDSS LMWDs overlaid on tracks of constant mass (in units of $M_{\odot}$ ) from Serenelli et al. (2001) for $M=0.148$ and $0.160 M_{\odot}$ and from Althaus et al. (2001) for $M \geqslant 0.169 M_{\odot}$. A canonical 0.6 $M_{\odot}$ DA WD has $\log g=8$ and the observed peak in the temperature distribution for SDSS DAs is $\log T_{\text {eff }} \approx 4$. The tracks are color coded to indicate the age of an LMWD of a given mass, calculated by these authors from the end of mass transfer onto the WD companion. The solid data point is $\mathrm{J} 2049+0005$, identified as an A2 star by Kilic et al. (2007b). The three WDs with $\log T_{\text {eff }}>4.4$ were not included in the Eisenstein et al. (2006) LMWD list.

(A color version of this figure is available in the online journal.)

MSP companions, their temperatures are very different. For example, the companion to MSP J1012+5307, whose mass of $0.16 \pm 0.02 M_{\odot}$ makes it the lowest mass confirmed WD in an MSP/WD system, has $T_{\text {eff }}=8550 \pm 25 \mathrm{~K}$ (van Kerkwijk et al. 1996), which falls in the range for previously cataloged pulsar WD companions, $3500 \mathrm{~K}<T_{\text {eff }}<15,000 \mathrm{~K}$, regardless of WD type and mass (Hansen \& Phinney 1998; van Kerkwijk et al. 2005). Indeed, none of the known He-core WD companions whose temperature has been measured are hotter than $T_{\text {eff }} \approx 10,000 \mathrm{~K}$. Our LMWDs all have temperatures above $8000 \mathrm{~K}$, and most have $T_{\text {eff }} \gtrsim 15,000 \mathrm{~K}$, indicating that we are looking at a younger population of objects than that found to date as MSP companions. ${ }^{11}$

A comparison to the evolutionary models produced by Serenelli et al. (2001) and Althaus et al. (2001) suggests that all but the lowest mass WD in our sample are younger than roughly $1 \mathrm{Gyr}$, and that all are younger than $5 \mathrm{Gyr}$ (see Figure 1). These ages are calculated from WD cooling models, and correspond to the time since the end of mass transfer onto the WD companions. By contrast, typical MSPs have ages of 5-10 Gyr; these are estimated from the pulsar characteristic (spin-down) age and, in a few cases, also from the companion WD's cooling age (e.g., J1012+5307; Lange et al. 2001).

\section{GREEN BANK TELESCOPE OBSERVATIONS}

The LMWDs were observed with the GBT over the course of five nights between 2006 January 12 and March 9. Each session began with a 1 minute observation of a test pulsar that was immediately analyzed to confirm a correct observing setup; the integration times for each target are listed in Table 1. At the central observing frequency of $820 \mathrm{MHz}$, the BerkeleyCaltech Pulsar Machine analog/digital filter bank (the design is described in Backer et al. 1997) provided $48 \mathrm{MHz}$ of bandwidth split into 96 spectral channels, for each of which we recorded total power samples every $72 \mu \mathrm{s}$. The resulting data were analyzed using standard pulsar search techniques

\footnotetext{
11 Because their colors are similar to those of main-sequence stars, WDs with $T_{\text {eff }} \lesssim 5000 \mathrm{~K}$ are unlikely to be targeted for/discovered via SDSS spectroscopy (Kilic et al. 2006).
} 
Table 1

SDSS LMWDs Observed with the GBT

\begin{tabular}{|c|c|c|c|c|c|c|c|c|}
\hline SDSS J & $\begin{array}{c}\text { SDSS } g \\
(\mathrm{mag})\end{array}$ & $\begin{array}{l}T_{\text {eff }} \\
(\mathrm{K})\end{array}$ & $\begin{array}{l}M_{\mathrm{WD}} \\
\left(M_{\odot}\right) \\
\end{array}$ & $\begin{array}{l}P_{\text {orb }} \\
(\mathrm{d})\end{array}$ & $\begin{array}{l}\text { Distance } \\
(\mathrm{kpc})\end{array}$ & $\begin{array}{l}b \\
\left(^{\circ}\right) \\
\end{array}$ & $\begin{array}{l}\text { Dispersion Measure } \\
\left(\mathrm{cm}^{-3} \mathrm{pc}\right)\end{array}$ & $\begin{array}{l}\text { Integration } \\
\text { Time (s) }\end{array}$ \\
\hline $084910.13+044528.7$ & $19.31 \pm 0.02$ & $9962 \pm 165$ & $\sim 0.169$ & $\lesssim 0.1$ & 1.5 & +28.3 & 112 & 3000 \\
\hline $123410.37-022802.9$ & $17.87 \pm 0.02$ & $17114 \pm 227$ & $0.18-0.19$ & $\widetilde{\curvearrowright}_{0} 0.3$ & 0.7 & +60.1 & 64 & 13400 \\
\hline $105353.89+520031.0$ & $18.93 \pm 0.02$ & $15399 \pm 400$ & $<0.196$ & $\widetilde{\Sigma}_{0.3}$ & 1.2 & +56.8 & 64 & 2400 \\
\hline $082212.57+275307.4$ & $18.33 \pm 0.01$ & $8777 \pm 40$ & $\sim 0.196$ & $\sim 0.3$ & 0.9 & +31.1 & 128 & 1800 \\
\hline $143633.29+501026.8$ & $18.23 \pm 0.01$ & $16993 \pm 229$ & $\sim 0.196$ & $\sim 0.3$ & 0.9 & +59.5 & 64 & 1500 and 1800 \\
\hline $162542.10+363219.1$ & $19.36 \pm 0.02$ & $24913 \pm 936$ & $\sim 0.196$ & $\sim 0.3$ & 1.5 & +44.1 & 64 & 3900 \\
\hline $234536.47-010204.9^{\mathrm{a}}$ & $19.50 \pm 0.02$ & $30270 \pm 1127$ & $<0.242$ & $\sim 7.5$ & 1.6 & -59.5 & 64 & 2400 \\
\hline $002207.65-101423.5$ & $19.77 \pm 0.02$ & $19444 \pm 758$ & $<0.292$ & $\lesssim 30$ & 1.8 & -71.8 & 64 & 2400 \\
\hline $002228.45+003115.5$ & $19.23 \pm 0.05$ & $17355 \pm 394$ & $<0.292$ & $\lesssim 30$ & 1.4 & -61.5 & 64 & 2200 \\
\hline $142601.47+010000.2$ & $19.34 \pm 0.02$ & $16311 \pm 359$ & $<0.292$ & $\lesssim 30$ & 1.5 & +55.4 & 64 & 2400 \\
\hline $163030.58+423305.7$ & $19.02 \pm 0.01$ & $14854 \pm 359$ & $<0.292$ & $\lesssim 30$ & 1.3 & +43.4 & 80 & 1800 \\
\hline $225242.25-005626.6$ & $18.59 \pm 0.03$ & $20479 \pm 433$ & $<0.292$ & $\widetilde{\lesssim} 30$ & 1.0 & -50.0 & 80 & 2400 \\
\hline $105611.03+653631.5$ & $19.77 \pm 0.03$ & $20112 \pm 634$ & $\sim 0.31$ & $\sim 35$ & 1.8 & +47.5 & 80 & 10400 \\
\hline $131033.25+644032.8^{\mathrm{a}}$ & $18.30 \pm 0.02$ & $39654 \pm 874$ & $\lesssim 0.360$ & $\sim 200$ & 0.9 & +52.3 & 64 & 1300 \\
\hline $163800.36+004717.7^{\mathrm{a}}$ & $18.84 \pm 0.01$ & $73149 \pm 4459$ & $\widetilde{\jmath}_{0.406}$ & $\sim 300$ & 1.2 & +29.7 & 112 & 1800 \\
\hline $204949.78+000547.3^{b}$ & $19.66 \pm 0.03$ & $8660 \pm 144$ & $<0.169$ & $\lesssim 0.1$ & 1.7 & -26.0 & 128 & 1860 \\
\hline
\end{tabular}

Notes. $g$ (PSF) magnitudes are from SDSS DR6. $P_{\text {orb }}$ is estimated by comparison to models from Tauris \& Savonije (1999); see also van Kerkwijk et al. (2005). Distances are calculated assuming a typical $M_{g}=8.5$. The listed DM is the maximum value used when searching for pulsations; it corresponds approximately to twice the maximum value obtained in the direction of each object with the Cordes \& Lazio (2002) model (for computing purposes the maximum DM is a multiple of 16).

a Not included in Eisenstein et al. (2006) catalog.

${ }^{\mathrm{b}}$ Kilic et al. (2007b) identify J2049+0005 as an A2 star.

implemented in the PRESTO package (Ransom 2001) installed in our computer cluster. This analysis was similar to that described in Camilo et al. (2006), with appropriate ranges of trial dispersion measure (DM) to account for dispersive interstellar propagation, and with substantial acceleration correction to partially account for the changing pulsar period caused by putative orbital motion.

We calculated the maximum DM expected in the direction of each target using the Cordes \& Lazio (2002) model for the distribution of free electrons in the Galaxy. A typical value for our high Galactic latitude targets was $\approx 40 \mathrm{~cm}^{-3} \mathrm{pc}$. In order to account for possible uncertainties in the model, we dedispersed the data up to a DM limit twice that obtained from the model. These values are given for each target in Table 1.

The orbital motions of the putative pulsars in these systems could significantly affect the apparent pulsar spin period, particularly for the companions to the lowest mass WDs in our sample (known MSP/WD binaries have orbital periods that range from a few hours to many months). In Table 1, we include rough estimates for binary periods based on comparisons to the models of Tauris \& Savonije (1999). Tauris \& Savonije (1999) calculated orbital properties for 1-2 $M_{\odot}$ companions to $1.3 M_{\odot}$ accreting NSs, and their models are consistent with the orbital periods found for systems with less massive companions (van Kerkwijk et al. 2005). While for $90 \%$ of known pulsars the maximum orbital acceleration is $\leqslant|25| \mathrm{m} \mathrm{s}^{-2}$ (Manchester et al. 2005; van Leeuwen et al. 2007), for $40 \%$ of our targets the estimated accelerations are $\gtrsim 100 \mathrm{~m} \mathrm{~s}^{-2}$. This significantly increases the parameter space that needs to be searched for pulsed signals and hence the computing time on the cluster, and also impacts our sensitivity limits (see discussion in Section 4.2).

\section{DISCUSSION}

No convincing pulsar signal was detected in our data. Below, we discuss the limitations of our search.

\subsection{Luminosity Sensitivity}

Models for WDs in the mass range of interest here predict absolute magnitudes of $M_{g} \sim 8.5$ (Eisenstein et al. 2006). We use SDSS Data Release 6 photometry (DR6; AdelmanMcCarthy et al. 2008) to obtain rough distance estimates to our LMWDs (see Table 1).

Luminositywise, our searches were extremely constraining. In order to calculate the minimum detectable period-averaged flux density, we use the standard modifications to the radiometer equation. We consider a pulsar duty cycle of $20 \%$ (fairly typical of MSPs) and compute sensitivity, conservatively, for DM = $50 \mathrm{~cm}^{-3}$ pc. At $820 \mathrm{MHz}$, the GBT gain is $2 \mathrm{~K} \mathrm{Jy}^{-1}$ and the system temperature is $25 \mathrm{~K}$. The sky temperature varies with location, but at the relevant high latitudes at this frequency it only adds a few Kelvin to the overall temperature. We consider an effective threshold signal-to-noise ratio of 10 . For an integration time of $2400 \mathrm{~s}$ (see Table 1), the sensitivity limit for long-period pulsars is $0.16 \mathrm{mJy}$. Pulsar luminosities are often measured at $1400 \mathrm{MHz}$; using a typical spectral index of -1.7 , the limiting sensitivity at that frequency is about $S_{1400}=0.06 \mathrm{mJy}$. For an MSP period of $3 \mathrm{~ms}$, our sensitivity at $1400 \mathrm{MHz}$ was roughly $50 \%$ worse, or $0.1 \mathrm{mJy}$ (and it quickly degrades for shorter periods, being three times worse for $1 \mathrm{~ms}$ ). Twelve of our targets fall within the FIRST footprint; none are detected in these data, for which the sensitivity limit is roughly $1 \mathrm{mJy}$ (Becker et al. 1995).

About $95 \%$ of MSPs known in the Galactic disk have luminosities $L_{1400} \equiv S_{1400} d^{2}>0.1 \mathrm{mJy} \mathrm{kpc}^{2}$, and some $90 \%$ have $L_{1400}>0.5 \mathrm{mJy} \mathrm{kpc}^{2}$. Since our targets have typical estimated distances of $1-2 \mathrm{kpc}$, our $L_{1400}$ limits for $3 \mathrm{~ms}$ periods ranged over $\approx 0.1-0.4 \mathrm{mJy} \mathrm{kpc}^{2}$. As far as luminosity alone is concerned, therefore, we should have been able to detect approximately 90\% of known MSPs had they been present in our targets and beaming radio waves toward the Earth. 


\subsection{Acceleration Sensitivity}

As mentioned earlier, if the lowest mass WDs in our sample are in binaries with NSs, these should be compact systems with orbital periods on the order of a few hours to a few days (Tauris \& Savonije 1999; van Kerkwijk et al. 2005). As a result, pulse smearing due to orbital motions could occur, severely complicating our search. To gauge the extent of this effect, we have calculated orbital accelerations for a number of systems with LMWDs in orbit around a $1.4 M_{\odot}$ NS.

When conducting an accelerated pulsar search with PRESTO, the free parameter related to the maximum acceleration searched actually specifies the maximum drift of Fourier components that is corrected. Relating this to a physical acceleration depends on the integration time and on the putative pulsar period. We consider a period of $3 \mathrm{~ms}$. For our integration times and computing resources, it was impracticable to fully correct for putative acceleration such as might be produced in systems with orbital periods of about $8 \mathrm{hr}$ or less. For example, for J0849+0445, an $M \sim 0.169 M_{\odot}$ WD that we observed for $3000 \mathrm{~s}$, the search sensitivity to a $3 \mathrm{~ms}$ pulsar was limited to a maximum acceleration of $60 \mathrm{~m} \mathrm{~s}^{-2}$. Because this search alone required several days of computing time on our 16-node cluster, it simply was not feasible to extend it to the maximum accelerations predicted for a WD of its mass (e.g., $300 \mathrm{~m} \mathrm{~s}^{-2}$ if $P_{\text {orb }} \sim 3 \mathrm{hr}$ and $1200 \mathrm{~m} \mathrm{~s}^{-2}$ if $P_{\text {orb }} \sim 1 \mathrm{hr}$ ).

Furthermore, for the six WDs with $M \lesssim 0.169 M_{\odot}$, our integration times may represent a substantial fraction of the binary orbital period (e.g., as much as $50 \%$ in the case of SDSS J1234; see Table 1). In these cases, the assumption of a constant apparent acceleration used in our search breaks down, further limiting our ability to detect a pulsed signal from these targets.

Nevertheless, we were still very sensitive to most kinds of known MSP/WD systems: essentially none are known in the disk of the Galaxy with $P_{\text {orb }}<14 \mathrm{hr}$. In summary, while we were not very sensitive to new kinds of exotic MSPs (e.g., with $P<1.5 \mathrm{~ms}$, or with $P_{\text {orb }}<12 \mathrm{hr}$ ), we should have detected some ordinary MSPs if they were orbiting a substantial fraction of our targets.

\subsection{Constraining the Number of LMWD/MSP Systems}

van Leeuwen et al. (2007) conducted a similar search for radio pulsations at $340 \mathrm{MHz}$ from unseen companions to eight SDSS LMWDs using the GBT. None of these observations contained signals warranting follow-up.

The eight WDs targeted by van Leeuwen et al. (2007), described in Liebert et al. (2004), were identified as candidate LMWDs based on initial model fits to SDSS spectra done by Kleinman et al. (2004). However, of the eight candidates, three are no longer considered LMWDs based on more recent analysis of their spectra by Eisenstein et al. (2006). ${ }^{12}$ We therefore updated the analysis performed by van Leeuwen et al. (2007) in order to place more stringent constraints on the likelihood $P_{\text {MSP }}$ that a given LMWD has an MSP companion.

van Leeuwen et al. (2007) assumed that a nondetection was the most likely outcome of their observations and estimated $P_{\text {MSP }}$ by using

$$
\left(1-P_{\text {beam }} \times P_{L} \times P_{\text {acc }} \times P_{\text {eff }} \times P_{\mathrm{MSP}}\right)^{N}>\frac{1}{2},
$$

where $P_{\text {beam }}$ is the MSP beaming fraction, $P_{L}$ is the luminosity completeness of the search, $P_{\text {acc }}$ is the sensitivity to the systems'

\footnotetext{
12 These three WDs are SDSS J081136.34+461156.4, J102228.02+020035, and $\mathrm{J} 130422.65+012214.2$.
}

accelerations, $P_{\text {eff }}$ is the search-algorithm success rate, and $N$ is the number of observed LMWDs. van Leeuwen et al. (2007) find that $P_{\mathrm{MSP}}<18 \pm 5 \%$; this limit rises to $26 \%$ if one removes the three objects no longer classified as LMWDs.

We use several of the same assumptions as van Leeuwen et al. (2007) in estimating $P_{\text {MSP. We }}$ set $P_{\text {beam }}=0.7 \pm 0.2$ and chose $P_{\text {eff }}=0.8$, a conservative estimate of our MSPdetection algorithm's success rate. Our luminosity sensitivity is $P_{L}=0.9$ and our acceleration sensitivity is $P_{\text {acc }}=0.9$; these two values reflect our confidence that our observations were sensitive enough to detect most MSPs with luminosities, orbital characteristics, and rotation periods comparable to those of the known population. We then find that $P_{\mathrm{MSP}}<10_{-2}^{+4} \%$.

\section{CONCLUSION}

We conducted a search for pulsar companions to 15 LMWDs at $820 \mathrm{MHz}$ with the GBT. No convincing pulsar signal was detected in our data. This is consistent with the findings of van Leeuwen et al. (2007), who conducted a search for radio pulsations at $340 \mathrm{MHz}$ from unseen companions to eight SDSS LMWDs (three of which are no longer considered LMWDs). However, our nondetections place stronger constraints on the probability that the companion to a given LMWD is a radio MSP. Our observations allow us to lower this likelihood from $<26 \%$ to $<10_{-2}^{+4} \%$.

Any radio search for pulsars is inherently biased, and even a completely unbiased search could not preclude the existence of MSPs in these systems. MSP beaming fractions are thought to be about $70 \% \pm 20 \%$, and therefore in some sensitive searches MSPs have not been detected even though there is strong evidence for their presence (e.g., the companion to the young pulsar J1906+0746; Lorimer et al. 2006). Given that NS blackbody emission is gravitationally bent, allowing us to view $>75 \%$ of the NS surfaces in X rays (Beloborodov 2002), X-ray observations could yet detect MSPs that might exist in these systems. None of these LMWDs have been observed since the ROSAT All-Sky Survey (Voges et al. 1999, 2000), and none were detected in that survey.

If LMWD companions are not NSs, the likelihood is that their currently unseen companions are also (very faint) WDs. Two dozen WD/WD binaries are known; in 10 systems both WD masses have been measured (see, Nelemans et al. 2005, and references therein). WD0957-666 has $M_{1}=0.37$ and $M_{2}=$ $0.32 M_{\odot}$; WD1101+364 has $M_{1}=0.29$ and $M_{2}=0.35 M_{\odot}$. The other WDs in these systems have $M \geqslant 0.35 M_{\odot}$. In the 14 other binaries discussed by Nelemans et al. (2005), only one of the WD masses is measured, with the companion WD mass given as a lower limit. Four of these systems harbor a WD with $0.23 \leqslant M<0.35 M_{\odot}$. If the SDSS LMWDs are confirmed as WD/WD systems, they will represent a significant addition to this population-and in a mass range that is still poorly sampled.

We thank Scott Ransom and the observing specialists at the GBT for their assistance and Kurtis Williams and Craig Heinke for helpful discussions. M.A.A. is supported by an NSF Astronomy and Astrophysics Postdoctoral Fellowship under award AST-0602099.

The Robert C. Byrd Green Bank Telescope is operated by the National Radio Astronomy Observatory, which is a facility of the US National Science Foundation operated under cooperative agreement by Associated Universities, Inc. We gratefully acknowledge GBT grant GSSP06-0008 for support of portions of our program. 
Funding for the SDSS and SDSS-II has been provided by the Alfred P. Sloan Foundation, the Participating Institutions, the National Science Foundation, the US Department of Energy, the National Aeronautics and Space Administration, the Japanese Monbukagakusho, the Max Planck Society, and the Higher Education Funding Council for England. The SDSS Web site is http://www.sdss.org/.

The SDSS is managed by the Astrophysical Research Consortium for the Participating Institutions. The Participating Institutions are the American Museum of Natural History, Astrophysical Institute Potsdam, University of Basel, University of Cambridge, Case Western Reserve University, University of Chicago, Drexel University, Fermilab, the Institute for Advanced Study, the Japan Participation Group, Johns Hopkins University, the Joint Institute for Nuclear Astrophysics, the Kavli Institute for Particle Astrophysics and Cosmology, the Korean Scientist Group, the Chinese Academy of Sciences (LAMOST), Los Alamos National Laboratory, the Max-PlanckInstitute for Astronomy (MPIA), the Max-Planck-Institute for Astrophysics (MPA), New Mexico State University, Ohio State University, University of Pittsburgh, University of Portsmouth, Princeton University, the United States Naval Observatory, and the University of Washington.

\section{REFERENCES}

Adelman-McCarthy, J. K., et al. 2006, ApJS, 162, 38

Adelman-McCarthy, J. K., et al. 2008, ApJS, 175, 297

Althaus, L. G., et al. 2001, MNRAS, 323, 471

Backer, D. C., et al. 1997, PASP, 109, 61

Becker, R. H., et al. 1995, ApJ, 450, 559
Beloborodov, A. M. 2002, ApJ, 566, L85

Benvenuto, O. G., \& De Vito, M. A. 2005, MNRAS, 362, 891

Camilo, F., et al. 2006, ApJ, 637, 456

Cordes, J. M., \& Lazio, T. J. W. 2002, arXiv:astro-ph/0207156

Driebe, T., Schoenberner, D., Bloecker, T., \& Herwig, F. 1998, A\&A, 339, 123

Eisenstein, D. J., et al. 2006, ApJS, 167, 40

Hansen, B. M. S., \& Phinney, E. S. 1998, MNRAS, 294, 569

Hansen, B. M. S., et al. 2007, ApJ, 671, 380

Kilic, M., et al. 2006, AJ, 131, 582

Kilic, M., et al. 2007a, ApJ, 671, 761

Kilic, M., et al. 2007b, ApJ, 660, 1451

Kleinman, S. J., et al. 2004, ApJ, 607, 426

Koester, D., Schulz, H., \& Weidemann, V. 1979, A\&A, 76, 262

Lange, C., Camilo, F., Wex, N., Kramer, M., Backer, D. C., Lyne, A. G., \& Doroshenko, O. 2001, MNRAS, 326, 274

Liebert, J., et al. 2004, ApJ, 606, L147

Liebert, J., et al. 2005, ApJS, 156, 47

Lorimer, D. R. 2008, Living Rev. Relativ., 11, 8

Lorimer, D. R., et al. 2006, ApJ, 640, 428

Manchester, R. N., Hobbs, G. B., Teoh, A., \& Hobbs, M. 2005, AJ, 129, 1993

Marsh, T. R., et al. 1995, MNRAS, 275, 828

Nelemans, G., et al. 2005, A\&A, 440, 1087

Panei, J. A., Althaus, L. G., Chen, X., \& Han, Z. 2007, MNRAS, 382, 779

Ransom, S. M. 2001, PhD thesis, Harvard Univ.

Serenelli, A. M., Althaus, L. G., Rohrmann, R. D., \& Benvenuto, O. G. 2001, MNRAS, 325, 607

Tauris, T. M., \& Savonije, G. J. 1999, A\&A, 350, 928

Tauris, T. M., \& van den Heuvel, E. P. J. 2006, in Compact Stellar X-Ray Sources, ed. W. Lewin \& M. van der Klis (Cambridge: Cambridge Univ. Press), 623

van Kerkwijk, M. H., et al. 1996, ApJ, 467, L89

van Kerkwijk, M. H., et al. 2005, in ASP Conf. Ser. 328, Binary Radio Pulsars, ed. F. A. Rasio \& I. H. Stairs (San Francisco, CA: ASP), 357

van Leeuwen, J., et al. 2007, MNRAS, 374, 1437

Voges, W., et al. 1999, A\&A, 349, 389

Voges, W., et al. 2000, VizieR Online Data Catalog, 9029, 0

York, D. G., et al. 2000, AJ, 120, 1579 